\title{
Secondary damage in trauma and limited access dressing: a review
}

\author{
Pramod Kumar $^{1}$, Akriti Gupta², Apoorva Gupta ${ }^{3}$ \\ ${ }^{1}$ Consultant Plastic Surgeon, King Fahd Central Hospital, Jazan 82666, Saudi Arabia. \\ ${ }^{2}$ Resident Physician (Pathology), University of Virginia, Charlottesville, VA 22903, USA. \\ ${ }^{3}$ Physician (Internal Medicine), Asante Rogue Regional Medical Center, Medford, OR 97504, USA.
}

Correspondence to: Dr. Pramod Kumar, Department of Plastic Surgery, King Fahad Central Hospital, Jazan 82666, Saudi Arabia. E-mail: pkiumar86@hotmail.com

How to cite this article: Kumar P, Gupta A, Gupta A. Secondary damage in trauma and limited access dressing: a review. Plast Aesthet Res 2020;7:29. http://dx.doi.org/10.20517/2347-9264.2019.71

Received: 7 Dec 2019 First Decision: 21 Apr 2020 Revised: 25 Apr 2020 Accepted: 6 May 2020 Published: 18 Jun 2020

Science Editor: Raúl González-García Copy Editor: Jing-Wen Zhang Production Editor: Jing Yu

\begin{abstract}
Secondary damage in trauma may increase morbidity, mortality and the cost of treatment considerably. This article reviews the literature of 46 relevant articles on this topic. We hope to provide a better understanding of the various mechanisms that can lead to secondary damage following major trauma and aim to improve the management of such in trauma patients. We also explore the utility of limited access dressing and its ability to minimize and treat secondary musculoskeletal trauma. Four interdependent cellular mechanisms have been described that contribute and perpetuate secondary tissue damage - lysosomal, protein/enzyme denaturation, membrane permeability and mitochondrial. Systemic changes are mainly due to systemic hypoxia and the systemic inflammatory response syndrome. Limited access dressing appears to be an efficient and cost-effective method for the management of secondary damage, as evidenced by the reduced number of debridements, shorter wound coverage time, and reduction in total length of hospital stay while lowering treatment costs and improving quality of care.
\end{abstract}

Keywords: Trauma, secondary damage, limited access dressing

\section{INTRODUCTION}

Cells are complex interconnected systems that work together to maintain a well-regulated microenvironment that is indispensable for their survival. Trauma to a single cell can affect overall homeostasis 
of the immediate environment. The extent of injury and tissue characteristics determine the extent of biochemical damage to adjacent cells, leading to cell death and tissue dysfunction. Secondary damage in trauma can increase patient morbidity, mortality and the cost of treatment considerably. A better understanding and control of secondary damage is thus essential for improved outcomes in trauma patients, which will, in turn, reduce morbidity and render management cost-efficient. In this article, we review the existing literature and analyze possible areas where standard medical and surgical intervention along with wound management using Limited Access Dressing (LAD) could lead to better outcomes.

\section{METHOD}

Secondary damage in trauma patients was defined. We looked up the relevant literature by using "Secondary Damage in Trauma" and "Limited Access Dressing" on PubMed and Google search engines. Relevant articles from molecular biology, physiology and pathophysiology were also reviewed to explain the mechanism of secondary damage in trauma. A total of 46 relevant articles were reviewed analyzed to find areas where standard medical and surgical intervention, along with wound management using LAD, could lead to better outcomes.

\section{Definition}

secondary injury, in simple words "by-stander" damage, of cells result from a secondary insult with destructive and biochemical mechanisms triggered by the mechanical destruction of tissue following direct trauma (the primary insult). Secondary damage may develop immediately (within hours) following the primary insult, and last from a few days up to weeks after. For instance, necrosis of peripheral tissues surrounding the damaged tissue can develop late after crush injuries ${ }^{[1]}$. Cells or tissue with ultra-structural damage at the time of trauma may die within hours to a few days following trauma. It is still controversial however, whether such cell death should be included under primary or secondary damage ${ }^{[2]}$. Secondary damage may also involve local or distant cells/tissues. From a biomedical point of view, it can occur due to hemorrhagic shock, compartment syndrome and/or ischemic necrosis ${ }^{[1,2]}$.

\section{Mechanism of secondary damage}

Secondary damage to local tissue: This was explained by Knight's Sport Injury Model $(1970)^{[2]}$ that was later updated based on improved biochemical understanding of tissue damage.

Secondary damage to distant tissue: This mainly occurs due to systemic hypoxia following hemorrhage or due to pro-inflammatory cytokines following systemic inflammatory response syndrome (SIRS) ${ }^{[3]}$.

\section{Mechanism of local secondary tissue injury}

Knight's sport injury model (1970)

Knight's Sport Injury Model (Secondary Injury Model) was first described in the mid 70's to account for the series of events that occur following injury in athletes ${ }^{[2,4]}$. This model effectively explains the possible mechanism that is triggered due to the loss of cellular hemostasis occurring in adjacent, uninjured tissues following primary injury and cell death.

It could be speculated that the outcomes of a primary insult/trauma is immediate cell death or permanent ultra-structural changes that lead to death of injured cells over time. The physiological response to cell death, in theory, could affect the functionality of uninjured cells. The physiologic stress leading to tissue damage is called a secondary injury.

Knight hypothesized that secondary injuries are primarily caused by two mechanisms: (1) hypoxic injury causing oncosis (cell swelling) and acidosis; and (2) enzymatic (lysosomal enzymes) injury. 
Hypoxic injury causing oncosis and acidosis

Secondary hypoxic injury occurs due to: hemorrhage, intravascular thrombosis, reduced blood flow from inflammation following increased viscosity, increased intravascular pressure following hematoma and muscle spasm.

Causes of secondary hypoxic injury are (1) anaerobic respiration - depending upon the susceptibility of the involved tissues to hypoxia, glycolic pathways of ATP production lasts from few minutes to $6 \mathrm{~h}$. During aerobic respiration, ATP production is hampered, and acidosis occurs; (2) ATP dependent $\mathrm{Na}^{+} \mathrm{K}^{+}$ATPase pump failure ${ }^{[5]}-\mathrm{Na}^{+}$and $\mathrm{Ca}^{2+}$ influx due to ATP dependent $\mathrm{Na}^{+}$pump failure leads to cellular homeostatic mechanism failure, leading to oncosis, cell death and necrosis.

Hypoxia leads to reduced ATP production. Hence, initial cryotherapy in musculoskeletal trauma is beneficial, as cold helps reduce ATP demand.

\section{Enzymatic (lysosomal) injury}

Enzymes released from lysosomes of the injured and dead tissues: (1) acid hydrolases and phospholipases: these enzymes lyse the cell membrane by cleaving the hydrocarbon chain of membrane phospholipids; and (2) proteases: proteases inactivate protein by cleaving peptide bonds.

Effect of enzyme on tissues: acid hydrolases, phospholipase and proteases can cause loss of cell membrane integrity and lead to increased hydropic swelling (oncosis) followed by cell death.

Also, trauma leads to inflammation and neutrophils are acute inflammatory cells and the first line of cellular defense. Human neutrophil proteins (defensins; HNP-1 to 3) are bactericidal agents and interfere with the function of smooth muscle cells in vessels, are prothrombotic, and may inhibit angiogenesis ${ }^{[6]}$.

\section{Updated secondary local tissue injury model}

Mechanism of secondary ischemic injury to local tissue

The updated secondary injury model is based on improved biochemical understanding of tissue damage. It has now been proven that ischemia resulting from vascular injury, rather than hypoxia, plays a more significant role in driving secondary damage. Hypoxia poses only one problem of inadequate oxygen supply, whereas ischemia presents with three possible consequences ${ }^{[2]}:(1)$ hypoxia - leads to anaerobic respiration and thus reduced ATP (adenosine triphosphate); (2) low glucose supply to tissues, leading to reduced ATP; and (3) acidosis that causes reduced cellular ability to produce ATP.

Hypoxia also affects mitochondrial function and leads to neural tissue damage as early as within $4 \min ^{[5]}$, and musculoskeletal injury in approximately three hours ${ }^{[7]}$.

\section{Mechanism for secondary direct cell injury to local tissue}

There are four possible mechanisms for secondary direct cell injury to local uninjured tissues: (1) lysosomal mechanisms (due to the release of destructive enzymes that require low $\mathrm{pH}$ for activation); (2) protein/ enzyme denaturation mechanisms (due to low $\mathrm{pH}$ ); (3) membrane permeability mechanisms (due to failure of sodium-potassium-ATPase pump) - this leads to cellular swelling; and (4) mitochondrial mechanisms (due to power house failure).

All these mechanisms are basically due to two types of changes - hypoxic and enzymatic.

\section{Lysosomal mechanisms}

Lysosomes, the main digestive organelle in a eukaryotic cell, is packed with catabolic enzymes maintained at an acidic intraluminal $\mathrm{pH}$. Under physiological conditions, these highly destructive enzymes, such as 
lipases, proteases and cathepsins, are contained within the lysosomal complex by the lysosomal membrane. In theory, externalization of lysosomal enzymes to the neutral $\mathrm{pH}$ of the cytoplasm would render these enzymes inactive. However, it is now known that a few forms of cathepsins remain active even at neutral $\mathrm{pH}$ and can trigger a cascade of events that lead to cell death from the lysosomal pathway ${ }^{[8]}$. The acidity of the local microenvironment can further activate the lysosomal enzymes released due to lysosomal damage.

There are various stimuli that can lead to increased permeability of the lysosomal membrane and externalization of catalytic enzymes. Lysosomal membrane permeabilization may be caused by various stimuli but the most common mechanism is by destruction of the lipid organization and oxidative damage to membrane bound proteins. Ischemic-reperfusion injury following acute trauma may lead to oxidative stress and membrane damage and destabilization from reactive oxygen species. The hydroxyl radical can cause lipid peroxidation and damage to membrane bound proteins. In addition, reactive oxygen species may also contribute by altering the lysosomal mechanism and activating calcium channels ${ }^{[9]}$.

By releasing highly destructive enzymes (e.g., phospholipase A, cathepsin B) following trauma, cell membranes and cellular proteins are damaged. Lysosomal enzymes cause intracellular cell death when released but when the enzymes become extracellular, secondary damage to adjacent tissue occurs. The normal extracellular $\mathrm{pH}$ (approximately 7.2) hampers the action of the majority of lysosomal enzymes but acidic environments $(\mathrm{pH} \leq 5)$ provide optimal conditions for their functioning. Experimentally, it has also been shown that inhibitors of lysosomal proteolytic pathways produce beneficial effects on injured musculoskeletal tissue $e^{[2]}$.

\section{Protein/enzyme denaturation mechanisms}

Metabolic failure leads to low $\mathrm{pH}$ that can cause denaturation of cellular proteins (enzymes) ${ }^{[5]}$. Denaturation of enzymes leads to loss of cellular functionality with the end-result of cell death. Although denaturation seems like an ultimate step in cell death, multiple factors can cause denaturation. Also, a low $\mathrm{pH}$ activates many lysosomal enzymes, leading to secondary damage by enzymatic mechanisms. Hence, there is an overlap of enzymatic and denaturation mechanisms that act together to produce secondary damage.

\section{Membrane permeability mechanisms}

Changes in membrane permeability cause: (1) oncosis (cellular swelling) leading to cells bursting; and (2) failure of ion pumps (sodium-potassium-ATPase pump)/changes in voltage gradient and the resulting uncontrolled influx of ions $\left(\mathrm{Na}^{+}\right.$and $\left.\mathrm{Ca}^{2+}\right)$ leads to cellular death. Increased intracellular $\mathrm{Ca}^{2+}$ following $\mathrm{Ca}^{2+}$ influx causes activation of enzyme phospholipase, leading to disruption of the phospholipid membrane and cell death ${ }^{[10]}$.

\section{Mitochondrial mechanisms}

Metabolic failure through mitochondrial (power plant of the cell) damage ${ }^{[11]}$ is one of the leading causes of cell death. Mitochondrial failure resulting in insufficient ATP may trigger other lethal pathophysiological processes leading to cell death. This indicates overlap of various theories in this regard.

Causes of mitochondrial injury are: (1) hypoxic or ischemic injury, the oxidative production of ATP is reduced in hypoxia and becomes inadequate for mitochondrial or cellular homeostasis ${ }^{[2,5,7,1]}$. Hypoxia activates a number of phospholipases and proteases that cause progressive failure of mitochondrial ion pumps and damage to the mitochondrial membrane. Also, a number of (intracellular) proteins (stress proteins-heat shock protein 70 family; ubiquitin) that are expressed ${ }^{[12]}$ induce protein catabolism within the injured cells; (2) oxidative or reperfusion injury, post-hypoxia reperfusion ${ }^{[5,11,13]}$ produces enormous amounts of oxygen-derived free radicals that exceed normal antioxidant defenses and tissue damage results. Re-perfusion free radical damage of the mitochondrial membrane leads to cell powerhouse (mitochondria) 
failure. Vasodilatation and hyper perfusion caused by nitric oxide in the post-injury period may accentuate the reperfusion injury; and (3) calcium influx injury, intra-cellular calcium levels increase due to energy dependent $\mathrm{Na}^{+} \mathrm{K}^{+}$pump failure and thus, mitochondria act as calcium sinks ${ }^{[1,14,15]}$. Increased mitochondrial $\mathrm{Ca}^{2+}$ leads to activation of calcium-dependent proteases and phospholipases. Increased mitochondrial $\mathrm{Ca}^{2+}$ leads to opening of the permeability transition pore, and inner mitochondrial membrane channel. This results in free passage of small molecules, osmotic swelling leading to outer membrane rupture, ATP depletion and apoptotic mitochondrial cell death.

\section{Mechanism of systemic secondary tissue injury}

Systemic hypoxia: systemic hypoxia can cause tissue hypoxia of various organs/tissues. Cellular and tissue hypoxia may lead to shock and various types of complications ${ }^{[16]}$.

Delayed neurological changes in high voltage electric burns may be due to changes in the endothelium of small vessels supplying nervous tissues ${ }^{[17]}$. Kidney and heart muscle are damaged due to the release of various intracellular chemicals, hemoglobin and myoglobin ${ }^{[18]}$.

SIRS: follows major trauma, leading to a phase of inflammatory response which involves the synthesis of acute phase proteins by the liver, complement activation and the release of pro- (IL-1, TNF alpha, IL- 6, IL-8) and anti-inflammatory cytokines (IL-10, IL-13). If the balance between the pro- and anti-inflammatory cytokines is disrupted, it leads to the development of SIRS and increases morbidity and mortality ${ }^{[19-22]}$ [Figure 1].

The auto amplification of cytokine production following major trauma/surgery can occur due to overwhelming multiple organ dysfunction syndrome (MODS) and/or severe infection, leading to a cytokine storm $^{[23]}$ [Figure 2]. Infection with exaggerated inflammatory responses induce more cells that produce cytokines and act as catalysts to the cytokine storm ${ }^{[24,25]}$.

Very high level of circulatory pro-inflammatory cytokines, as in a cytokine storm, may cause uncontrolled, auto destruction that leads to life threatening MODS.

Immune depression: cytokines are known to cause immune depression in musculoskeletal trauma ${ }^{[26]}$.

Microvascular dysfunction and increased tissue pressure can occur following musculoskeletal trauma and lead to secondary soft tissue damage and compromised skeletal muscle function ${ }^{[27]}$.

\section{Clinical implications of secondary injury}

Repeated debridement and consequent exposure of vital structures - due to secondary injury following initial and surgical trauma, new necrosis occurs, requiring repeated debridement.

Complex reconstructive procedures are required on several occasions to cover vital exposed structures following repeated debridement.

SIRS - Pro-inflammatory cytokines are produced due to tissue damage that increase several times following infection, and if absorbed in large quantities, may produce SIRS, MODS and multiple organ failure.

Immobilization, edema and inflammation lead to functional loss and stiff joints ${ }^{[28]}$.

Missed injuries like spinal injuries may lead to serious crippling injuries ${ }^{[29]}$.

\section{Clinical strategy against secondary damage in trauma}

In the management of complex surgical problems, a surgeon has three options ${ }^{[30]}:$ (1) avoidance (laissezfaire); (2) aggressive approach; or (3) temporizing maneuvers. 


Major Trauma
Release of Pro-inflammatory Cytokines
Produced by monocytes and
macrophages

(IL-1, TNF- alpha and IL-6 are associated with pathological pain )

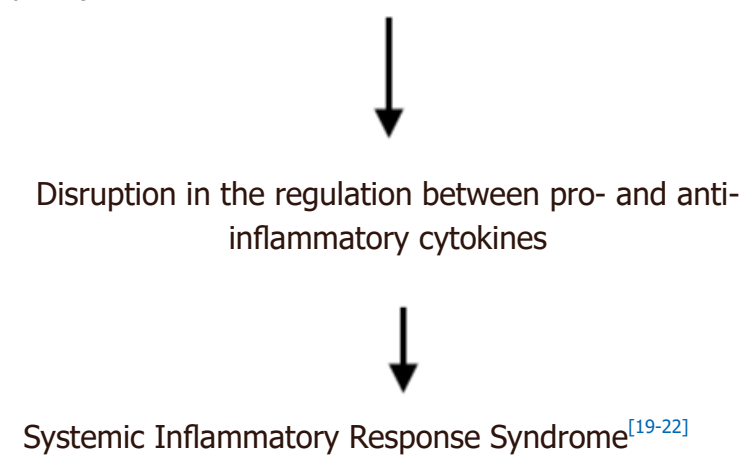

Figure 1. Pathogenesis of systemic inflammatory response syndrome

Avoidance leaves natural biochemical mechanism to overcome the damage but in contrast, the insult from an aggressive approach to counter secondary damage may aggravate the problem.

Hence, to counter secondary damage in trauma cases, the best clinical strategy is to employ temporizing maneuvers.

In temporizing maneuvers, damage control methodologies include: (1) quick action to stabilize a patient's local and systemic conditions; (2) prevention of further secondary damage; and (3) after the acute recovery phase is over, secondary definitive management is started.

Rapid and temporary stabilization is achieved by using LAD. Delaying the onset and progress of secondary damage has been attempted by using cryotherapy, but there is a lack of data related to the temperature and duration of cryotherapy required. Secondary, definitive reconstruction may be performed under LAD.

Cryotherapy (exposure to extreme cold) has been used to delay or prevent secondary injury in trauma or sports injury, but optimal tissue temperatures, the duration of cryotherapy, and the need for application pressure to minimize secondary injury following musculoskeletal trauma have yet to be established ${ }^{[31]}$. 


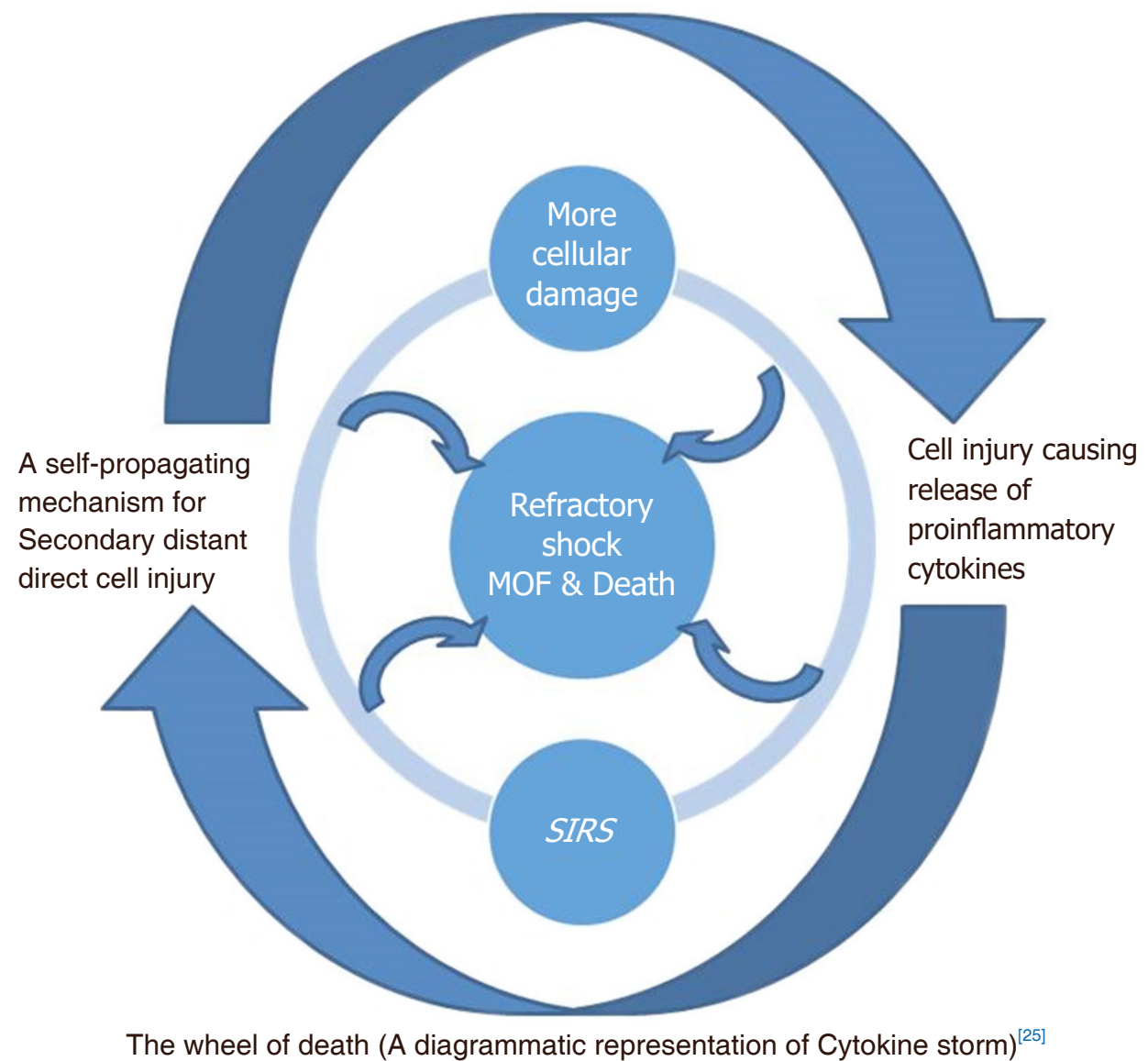

Figure 2. Diagrammatic representation of cytokine storm - the wheel of death. MOF: multiple organ failure; SIRS: systemic inflammatory response syndrome

Hyperbaric oxygen (HBO): in an experimental study on rats with contused calf muscle injury, following 7 days of treatment with $100 \%$ oxygen in a HBO chamber at 2.5 atmospheres pressure for 2 hours/day. $\mathrm{HBO}$ was found to reduce edema and inflammation, and accelerated myogenesis. This indicated beneficial effects of $\mathrm{HBO}$ against secondary damage. The mechanisms of healing following $\mathrm{HBO}$ therapy for muscle injuries have yet to be explained however ${ }^{[32]}$.

Anticytokine and antimediator therapy: has been tried in SIRS and MODS with limited success.

Forced diuresis and dialysis is recommended to prevent and treat renal dysfunction following myoglobinuria/hemoglobinuria ${ }^{[33]}$.

Neurological/neurosurgical management and rehabilitation of neurological secondary injury has been advised $^{[34,35]}$.

Advanced wound management using special modern dressing significantly reduces infection rates and the need for corrective surgeries ${ }^{[36]}$.

\section{LAD protocol in trauma}

LAD is applied over the affected part after saline wash. After surgical removal of obviously dead tissue, application of antimicrobial agents, and complete hemostasis, LAD is applied. Pressure bandaging and appropriate splints are applied as required. Continuous or intermittent negative pressure is applied as per 
protocol or as required. The minimum required effective negative pressure produces folds of plastic of LAD that lie snuggly over the wound or skin under LAD; higher pressures are guided by pain complaints by the patient and bleeding from the wound. Physiotherapy and occupational therapy may be started at the earliest. Obviously dead tissues are removed surgically and a new LAD is reapplied every 5-7 days. The LAD may be changed if significant leakage occurs. Daily LAD wash with saline and antimicrobial (Betadine) solution is performed. A secondary, elective procedure is performed (e.g., split skin graft/flap etc.) as and when required. Physiotherapy may be required after satisfactory healing ${ }^{[25,30,37]}$.

\section{Role of LAD}

LAD provides limited access of wound pathogens to the hospital environment and vice versa. LAD is helpful in preventing and treating secondary damage in trauma by reducing edema and removing harmful enzymes, toxins and other chemicals from the open wound ${ }^{[37]}$.

\section{Effects of LAD on oncosis (cellular swelling)}

Cellular swelling due to sodium and potassium pump failure obstructs the microcvasculature, leading to cell death and necrosis of the affected tissue. Intermittent negative pressure of LAD produces intermittent compression of the part under LAD leading to reduction in edema. This intra LAD compression, if combined with early intra LAD physiotherapy, effectively controls edema. Edema reduction thus improves the circulation of adjacent tissues ${ }^{[37]}$.

\section{Effects of LAD on $\mathrm{pH}$}

In a RCT (randomized control trial) ${ }^{[38]}, 42$ patients with chronic wounds in each group (LAD, conventional dressing) were studied for wound surface $\mathrm{pH}$. On the 10th post-operative day, the LAD group showed a significant $(P=0.048)$ reduction in $\mathrm{pH}$ as compared to the conventional dressing group (LAD group $0.41 \pm$ 0.26 vs. conventional group $0.83 \pm 0.52)$ with the mean wound surface $\mathrm{pH}( \pm \mathrm{SD})$ in the LAD group $7.5 \pm 0.43$.

Following trauma, the release of lysosomal enzymes is responsible for secondary damage. Lysosomal enzyme activity (lysosomal digestion) is optimal in acidic $\mathrm{pH}(<5)$, reduced at near neutral $\mathrm{pH}$, and is nearly de-activated at a $\mathrm{pH}$ of $7.2^{[39]}$. Lysosomal enzymes are not only removed by LAD but also deactivated, or its activity reduced by a change in $\mathrm{pH}$ under LAD. Hence, LAD may prove to be an effective tool to control secondary damage following trauma.

\section{$L A D$ provides a safe environment during waiting or temporization}

during the waiting period, infection and SIRS may pose a difficult problem but is controlled effectively with LAD.

1. Control of infection. LAD controls infection in the following ways ${ }^{[37,40]}$ : wound isolation and safe disposal of drainage. The important feature of LAD design helps to control infection.

Prevention of wound invasion: negative pressure of LAD provides an alternate channel for the movement of microorganisms. Intermittent or continuous negative pressure reduces bacterial concentrations $\left(<10^{5} / \mathrm{g}\right.$ tissue) to a level that prevents invasion.

Mechanical disruption of quorum sensing by negative pressure occurs as negative pressure prevents the desired concentration of bacterial chemicals through intermittent/continuous removal.

Mechanical disruption of biofilm: Higher intra-LAD negative pressure can cause disruption of the biofilm and expose bacteria in the niche environment to negative pressure. 
MDR (multi drug resistant) organisms can be effectively treated: Multi-drug resistant organisms are not resistant to the negative pressure of LAD.

\section{Control of SIRS}

LAD reduces systemic symptoms and signs of toxicity related to traumatized tissues, burns and gangrene. In a study of two groups comprising 54 burn patients (27 in each of LAD and control groups; at the time of induction, both groups showed no significant difference), there was no statistical difference in SIRS on day 1, but SIRS and organ dysfunction on day 5 was significantly lower ( $P$-values of 0.029 and 0.017 respectively) in the LAD treated group ${ }^{[30,37]}$.

\section{Protection of ischemia induced oxidative damage in LAD treated wounds}

Ischemia induced anaerobic respiration leads to reduced ATP production, and reduced antioxidant protection $^{[41]}$. Studies on LAD treated burn wounds ${ }^{[42,43]}$, diabetic wounds ${ }^{[44]}$ and chronic wounds $s^{[45,46]}$ have shown significant reduction in oxidative stress (malondialdehyde level), and significant increase in antioxidants and nitric oxide levels.

\section{Clinical study to find role of LAD in trauma}

In a case series ${ }^{[30]}$ of 20 consecutive cases of musculoskeletal extremity trauma treated with LAD without specific controls, 14 cases had exposed, problematic structures with exposed bone in 8 cases, exposed tendons in 3 cases, exposure of both bone and tendon in 2 cases, and an exposed injured brachial artery in 1. Results were quite encouraging. Edema under LAD in these cases was minimal. There was a reduction in the number of debridements: total number of debridement procedures was 23 in 20 patients (average 1.15/patient; range 0-3). Wound bed preparation time was excellent in 5/18 cases, fair in 11/18 cases and poor in $2 / 18$ cases. Excellent ( $>99 \%$ of grafted area) graft take was seen in 18/20 cases. Conversion rates from cases that required complex reconstructive procedures (e.g., flap) for exposed vital structures to simple reconstructive procedures (SSG) was $11 / 13 \times 100=84.6 \%$. Functional recovery of the hand was excellent in $4 / 10$ cases, fair in $2 / 10$ cases, and poor in $4 / 10$ cases.

The average cost of treatment was less than one-third of the treatment cost for similar procedures using wet-to-dry dressing (cost calculation was done based on reduced number of debridements, reduced anesthetic requirement, excellent graft take, reduced post-treatment physiotherapy, and rehabilitation costs).

From an administrative point of view, the quality of care was improved due to the reduction in required resources in emergency.

\section{CONCLUSION}

It was concluded that in addition to available medical and surgical interventions, substituting conventional closed dressings with LAD in cases of musculoskeletal trauma helps in reducing secondary damage as evidenced by the reduced number of debridements, reduced wound coverage time, and reduction in total length of hospital stay while lowering treatment costs and improving quality of care.

\section{DECLARATIONS}

\section{Authors' contributions}

Made substantial contributions to the conception and design of the study and performed data analysis and interpretation: Kumar P

Made substantial contributions to data acquisition and performed data analysis and interpretation: Gupta A, Gupta A 


\section{Availability of data and materials}

Not applicable.

\section{Financial support and sponsorship}

None.

\section{Conflicts of interest}

All authors declared that there are no conflicts of interest.

\section{Ethical approval and consent to participate}

Not applicable.

\section{Consent for publication}

Not applicable.

\section{Copyright}

(c) The Author(s) 2020.

\section{REFERENCES}

1. Bogens RB, Liu-Snyder P. Understanding Secondary Injury. Q Rev Biol 2012;87:89-127.

2. Merrick MA. Secondary injury after musculoskeletal trauma: a review and update. J Athl Train 2002;37:209-17.

3. Bone RC, Balk RA, Cerra FB, Dellinger RP, Fein AM, et al. Definitions for sepsis and organ failure and guidelines for the use of innovative therapies in sepsis. The ACCP/SCCM Consensus Conference Committee. American College of Chest Physicians/Society of Critical Care Medicine. Chest 1992;101:1644-55.

4. Knight KL. Cryotherapy in Sports Injury Management. Human Kinetics Chamain, IL: 1995. pp. 3-98

5. Banasik JL. Cell inury, aging and death. In: Costead LC, Banasik JL, editors. Pathophysiology: Biological and Behaorial Perspectives. 2nd ed. WB Saundrs Philadelhia, PA: 2000. pp. 76-91.

6. Kougias P, Chai H, Lin PH, Yao Q, Lumsden AB, et al. Defensins and cathelicidins: neutrophil peptides with roles in inflammation, hyperlipidemia and atherosclerosis. J Cell Mol Med 2005;9:3-10.

7. Belkin M, Brown RD, Wright JG, LaMorte WW, Hobson RW. A new quantitative spectrophotometric assay of ischemia-reperfusion injury in skeletal muscle. Am J Surg 1988;156:83-6.

8. Serrano-Puebla A, Boya P. Lysosomal membrane permeabilization in cell death: new evidence and implications for health and disease. Ann N Y Acad Sci 2016;1371:30-44.

9. Aits S, Jäättelä M. Lysosomal cell death at a glance. J Cell Sci 2013;126:1905-12.

10. Starke PE, Hoek JB, Farber JL. Calcium-dependent and calcium-independent mechanisms of irreversible cell injury in cultured hepatocytes. J Biol Chem 1986;261:3006-12.

11. Manjo G, Joris I. Cells, tissues, and disease: principles of general pathology. Blackwell Scientific; Cambridge, MA: 1996.

12. Kim SO, Baines CP, Critz SD, Pelech SL, Katz S, et al. Ischemia induced activation of heat shock protein 27 kinases and casein kinase 2 in the preconditioned rabbit heart. Biochem Cell Biol 1999;77:559-67.

13. Sabido F, Milazzo VJ, Hobson RW, Duran WN. Skeletal muscle ischemia-reperfusion injury: a review of endothelial cell-leukocyte interactions. J Invest Surg 1994;7:39-47.

14. Bernardi P. Mitochondria in muscle cell death. Ital J Neurol Sci 1999;20:395-400.

15. Rizzuto R, Bernardi, Pozzan T. Mitochondria as all round players of the calcium game. J Physiol 2000;529:37-47.

16. Kirkman E, Watts S. Haemodynamic changes in trauma. Br J Anaesth 2014;113:266-75.

17. Fish R. Electric shock, Part II: nature and mechanisms of injury. J Emerg Med 1993;11:457-62.

18. Lee RC. Injury by electrical forces: pathophysiology, manifestations, and therapy. Curr Probl Surg 1997;34:677-764.

19. Jaffer U, Wade RG, Gourlay T. Cytokines in the systemic inflammatory response syndrome: a review. HSR Proc Intensive Care Cardiovasc Anesth 2010;2:161-75.

20. Socha LA, Gowardman J, Silva D, Correcha M, Petrosky N. Elevation in interleukin 13 levels in patients diagnosed with systemic inflammatory response syndrome. Intensive Care Med 2006;32:244-50.

21. Zhang JM, An J. Cytokines, inflammation, and pain. Int Anesthesiol Clin 2007;45:27-37.

22. Bates P, Parker P, McFadyen I, Pallister I. Demystifying damage control in musculoskeletal trauma. Ann R Coll Surg Engl 2016;98:291-4.

23. Aikawa N. Cytokine storm in the pathogenesis of multiple organ dysfunction syndrome associated with surgical insults. Nihon Geka Gakkai Zasshi 1996;97:771-7. (in Japanese)

24. Chousterman BG, Swirski FK, Weber GF. Cytokine storm and sepsis disease pathogenesis. Semin Immunopathol 2017;39:517-28.

25. Kumar P. Wheel of death: cytokine cascade (cytokine storm) in cases with extensive wound. JSWCR 2013;6:1-2. 
26. Reikerås O. Immune depression in musculoskeletal trauma. Inflamm Res 2010;59:409-14.

27. Müller M, Disch AC, Zabel N, Haas NP, Schaser KD. Initial intramuscular perfusion pressure predicts early skeletal muscle function following isolated tibial fractures. J Orthop Surg Res 2008;3:14.

28. Abghari M, Monroy A, Schubl S, Davidovitch R, Egol K. Outcomes following low-energy civilian gunshot wound trauma to the lower extremities: results of a standard protocol at an urban trauma center. Iowa Orthop J 2015;35:65-9.

29. Metak G, Scherer MA, Dannöhl C. Missed injuries of the musculoskeletal system in multiple trauma--a retrospective study. Zentralbl Chir 1994;119:88-94. (in German)

30. Kumar PP, Sharma A. The limited access dressing for damage control in trauma patients. Wounds 2010;22:188-92.

31. Schaser KD, Stover JF, Melcher I, Lauffer A, Haas NP, et al. Local cooling restores microcirculatory hemodynamics after closed softtissue trauma in rats. J Trauma 2006;61:642-9.

32. Oyaizu T, Enomoto M, Yamamoto N, Tsuji K, Horie M, et al. Hyperbaric oxygen reduces inflammation, oxygenates injured muscle, and regenerates skeletal muscle via macrophage and satellite cell activation. Sci Rep 2018;8:1288.

33. Pezzi M, Giglio AM, Scozzafava A, Serafino G, Maglio P, et al. Early intensive treatment to prevent kidney failure in post-traumatic rhabdomyolysis: case report. SAGE Open Med Case Rep 2019;7:2050313X19839529.

34. Walter T, Schwabe P, Schaser KD, Maurer M. Positive outcome after a small-caliber gunshot fracture of the upper cervical spine without neurovascular damage. Pol J Radiol 2016;81:134-7.

35. Lammertse DP. Neurorehabilitation of spinal cord injuries following lightning and electrical trauma. NeuroRehabilitation 2005;20:9-14.

36. Abdel-Sayed P, Hirt-Burri N, de Buys Roessingh A, Raffoul W, Applegate LA. Evolution of biological bandages as first cover for burn patients. Adv Wound Care (New Rochelle) 2019;8:555-64.

37. Kumar P. Exploiting potency of negative pressure in wound dressing using limited access dressing and suction-assisted dressing. Indian J Plast Surg 2012;45:302-15.

38. Kumar P, Honnegowda T. Effect of limited access dressing on surface $\mathrm{pH}$ of chronic wounds. Plast Aesthet Res 2015;2:257.

39. Attaix D, Taillnadier D. The critical role of the ubiquitin-proteasome pathways in muscle wasting in comparison to lysosomal and $\mathrm{Ca}^{2+}$ dependent systems. In: Bitter EE, Rivert AJ, editors. Intracellular protein degradation. JAI Press; Greenwitch CT: 1998. pp. 235-66.

40. Kumar P. Limited access dressing and wound infection. Plast Aesthet Res 2015;2:237-8.

41. Wu MY, Yiang GT, Liao WT, Tsai AP, Cheng YL, et al. Current Mechanistic Concepts in Ischemia and Reperfusion Injury. Cell Physiol Biochem 2018;46:1650-67.

42. Honnegowda TM, Kumar P, Padmanabha Udupa EG, Sharan A, Singh R, et al. A comparative study to evaluate the effect of limited access dressing (LAD) on burn wound healing. Int Wound J 2016;13:791-8.

43. Honnegowda TM, Padmanabha Udupa EG, Rao P, Kumar P, Singh R. Superficial burn wound healing with intermittent negative pressure wound therapy under limited access and conventional dressings. World J Plast Surg 2016;5:265-73.

44. Honnegowda T, Kumar P, Prabhu K, Kumar A, Rao P, et al. A comparative study to evaluate the effect of limited access dressing on diabetic ulcers. Plast Aesthet Res 2015;2:266.

45. Honnegowda TM, Kumar P, Udupa P, Rao P, Bhandary S, et al. Effect of limited access dressing on hydroxyproline and enzymatic antioxidant status in nonhealing chronic ulcers. Indian J Plast Surg 2014;47:216-20.

46. Honnegowda TM, Kumar P, Padmanabha Udupa EG, Sharan A, Singh R, et al. Effects of limited access dressing in chronic wounds: A biochemical and histological study. Indian J Plast Surg 2015;48:22-8. 